\title{
Sepsis: the silent killer we should be stopping
}

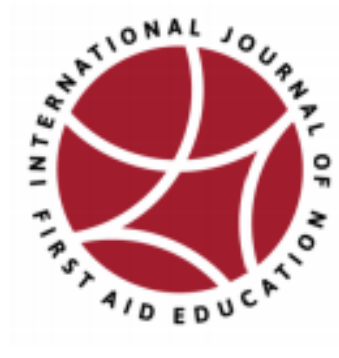

Dear Editors,

The UK Sepsis Trust estimates that sepsis may kill as many as six million people globally each year. We know that sepsis goes unrecognised by members of the public, and that often victims present to healthcare too late as a result. We also know that healthcare professionals could be better educated, so that sepsis diagnoses can be made more quickly, and treatment administered as fast as possible. Earlier identification and treatment could prevent many of the millions of unnecessary deaths every year. First Aid providers can help by equipping themselves with the information they need to recognise the signs of sepsis and seek medical attention urgently, thereby starting the Chain of Survival Behaviour (IFRC, 2016) as early as possible.

\section{What is sepsis?}

Sepsis is a reaction to infection in which the body attacks its own organs and tissues. It can arise in response to any infection, but most typically a bacterial infection of the lungs, urinary tract, skin/soft tissues (arising from a bite, cut or sting or from cellulitis) or abdomen (such as a perforated bowel). If not spotted and treated quickly, sepsis can rapidly lead to organ failure and death.

\section{The human and economic cost}

Sepsis is an indiscriminate condition, claiming young and old lives alike and affecting the previously fit and healthy. Approximately six million deaths (Fleishmann, et al, 2017) are caused by sepsis worldwide every year, but many of those could be saved by increased public awareness, education for healthcare professionals and other basic care provisions (Daniels, Nutbeam, \& McNamara, 2011). Data from a recent study commissioned by the UK Sepsis Trust and carried out by the independent York Health Economics Consortium (YHEC) found that there are likely to be at least 260,000 cases of sepsis annually in the UK - over 100,000 more than initial projections suggested (Whitewater Charitable Trust, 2017).

According to the report, the cost of sepsis is likely to be $£ 15.6$ billion every year for the UK economy, rather than the $£^{2.5}$ billion previously estimated (Vincent et al, 2006). However, government intervention to improve access to healthcare and reliability of basic care could make a huge impact, saving thousands of lives and reducing the cost to the National Health Service (NHS) by around £300 million. 


\section{Improving care globally}

Earlier this year, the World Health Organization (WHO) adopted a resolution on sepsis which makes the condition a global priority for policy makers and healthcare authorities. The Global Sepsis Alliance (GSA) Executive Committee helped to gather information from clinicians and families in over 70 countries, initiating a sepsis resolution which was unanimously recommended by the WHO's Executive Board and adopted at the 70th World Health Assembly in Geneva. The resolution means the new WHO Director-General, Dr Tedros, will draw attention to the public health impact of sepsis, and urge all 194 United Nations member states to develop action plans to help save lives and improve outcomes for survivors of sepsis in developed and developing countries. With first aid education intrinsic to public health, we urge first aid program managers to engage with the development of these plans.

The WHO has dedicated \$4.6 million USD of its budget to implementing the Resolution's recommendations, which include the effective use of antimicrobials, the introduction of affordable vaccines, treating sepsis as an emergency, providing suitable services for survivors, communicating with patients and relatives using the term 'sepsis' to improve public awareness and engaging in advocacy efforts, including those centered around World Sepsis Day (13th September). The adoption of this sepsis resolution is a crucial step in the right direction for countries all over the world which urgently require focus and resources to reclaim the millions of lives lost unnecessarily to sepsis each year. It also provides an important opportunity for first aid educators to work with policy makers on this issue.

\section{Improving care in the UK}

The new National Institute for Health and Care Excellence (NICE) sepsis guideline (nice.org.uk/guidance/ng51) - published in July 2016 and building on care recommendations developed by the UK Sepsis Trust - provides best practice guidance for healthcare professionals confronted with potential cases of sepsis. The guideline advises that sepsis be considered in any patient with an infection and that the condition should be treated with the same urgency as a heart attack. It describes the signs and symptoms that clinicians should check for, dividing these into high and moderate risk criteria and delineating where the person should be treated. It further advises which tests to use in diagnosing sepsis and monitoring response to therapy.

For lay responders, there are six symptoms to look out for, because sepsis can occur as the result of any infection. There could be indication of sepsis with even just one of these signs. This mnemonic was devised by the UK Sepsis Trust using a modified Delphi method among over 100 survivors.

Slurred speech or confusion

Extreme shivering or muscle pain 
Passing no urine (in a day)

Severe breathlessness

It feels like you're going to die

Skin mottled or discolored

First Aid providers have opportunity to advocate for the people in their care with symptoms of infection, by listening to the patient and their relatives when they express instincts that something might be seriously wrong. Phrases like "I've never seen him this ill" or "I feel like I'm going to die" should provide first aiders with a sense of urgency.

It's crucial that First Aid providers and instructors take steps to make Chain of Survival Behaviours for sepsis as effective as possible. Learning to recognise sepsis as part of all first aid education would mark crucial progress. It is also imperative that public health bodies take immediate steps to educate lay responders and other members of the public so that sepsis can be managed as efficiently as possible.

\section{Dr Ron Daniels BEM, CEO of UK Sepsis Trust}

\section{$\underline{\text { References }}$}

Daniels, R., Nutbeam, T., \& McNamara, G. (2011). The sepsis six and the severe sepsis resuscitation bundle: a prospective observational cohort study. Emergency Medicine Journal, 28(6): 50.

Fleischmann, C., Scherag, A., Adhikari, N.K.J., Hartog, C.S., Tsaganos, T., Schlattmann, P., . . Reinhart, K. (2015). Global burden of sepsis: a systematic review. Crit Care. 2015; 19(Suppl 1): P21. doi: $\underline{10.1186 / \mathrm{cc} 14101}$

International Federation of Red Cross and Red Crescent Societies (IFRC), (2016), International first aid and resuscitation guidelines 2016; Geneva, Switzerland.

Vincent, L., Sakr, M., Sprung, M., Ranieri, M., Reinhart, M., Gerlach, M., . . \& Payen, D. (2006). Sepsis in European intensive care units: Results of the SOAP study. Critical Care Medicine, 34(2), 344-353. 
Whitewater Charitable Trust (2017). The Cost of Sepsis Care in the UK. York, UK: Hex, N., Retzler, J., Bartlett, C., \& Arber, M. Retrieved from https://sepsistrust.org/wp-

content/uploads/2018/03/YHEC-Sepsis-Report-FINAL.pdf 